Abanico Veterinario. Enero-Diciembre 2020; 10:1-10. http://dx.doi.org/10.21929/abavet2020.39

Artículo Original. Recibido: 25/08/2020. Aceptado: 28/11/2020. Publicado: 20/12/2020. Clave:2020-73.

\title{
Efecto del nivel de dióxido de carbono de la incubadora sobre el desarrollo embrionario y parámetros de eclosión en pollo de engorda
}

\author{
Effect of incubator carbon dioxide level on embryonic development and hatching \\ parameters in broiler chicken
}

\section{Prado-Rebolledo Omar* ID, Castellano-Ortega José ID, Ruíz-Ramírez Johnatan ID, Zepeda-Batista José ID, García-Casillas Arturo** ID}

Facultad de Medicina Veterinaria y Zootecnia, Universidad de Colima. México. *Autor responsable: PradoRebolledo Omar. Kilómetro 40, Carretera Colima-Manzanillo. Crucero de Tecomán, Colima. C.P. 28100. **Autor de correspondencia: García-Casillas Arturo. omarpr@ucol.mx, jcastellanos4@ucol.mx, jruiz7@ucol.mx, jzepeda15@ucol.mx, cesargarciacasillas@hotmail.com

\section{RESUMEN}

El oxígeno $\left(\mathbf{O}_{2}\right)$ y el dióxido de carbono $\left(\mathbf{C O}_{2}\right)$ son gases vitales para el embrión durante el proceso de incubación, su nivel es imprescindible en el momento del picaje, con la finalidad de evaluar el efecto del nivel de dióxido de carbono de la incubadora sobre el desarrollo embrionario, los parámetros de eclosión y el posterior crecimiento del pollo de engorda, se midió la pérdida de humedad, incubabilidad, peso del pollo, tamaño del pollo, glucosa sanguínea, hematocrito y proteínas plasmáticas. Un total de 600 huevos de reproductora comercial Cobb 500 de 41 semanas, se seleccionaron por peso de 65 y $70 \mathrm{~g}$, se distribuyeron en dos máquinas incubadoras. Una máquina se mantuvo a 4000 ppm y la otra a 3000 ppm de $\mathrm{CO}_{2}$. Se utilizó un diseño factorial $2 \times 2$. La incubabilidad fue mayor a $3000 \mathrm{ppm}$ de $\mathrm{CO}_{2}$ y peso de huevo de $65 \mathrm{~g}$; el pollo más pesado fue con huevo de $70 \mathrm{~g}$, a mayor ppm de $\mathrm{CO}_{2}$ menor pérdida de humedad, a menor ppm de $\mathrm{CO}_{2}$ se observó un pollo más grande, los niveles de glucosa no se afectaron, pero los valores de proteínas plasmáticas fueron menores a $3000 \mathrm{ppm}$ de $\mathrm{CO}_{2}$. Se mejoran los parámetros de eclosión al bajar las ppm de $\mathrm{CO}_{2}$ durante el proceso de incubación.

Palabras clave: incubación, dióxido de carbono, embrión, gases.

\begin{abstract}
Oxygen $\left(\mathbf{O}_{2}\right)$ and carbon dioxide $\left(\mathbf{C O}_{2}\right)$ are vital gases for the embryo during the incubation process, its level is essential at pipping, to evaluate the effect of incubator carbon dioxide level on embryonic development, hatching parameters, and post-hatch growth of broiler, humidity loss, hatchability, weight of chicken, size of chicken, blood glucose, hematocrit and plasma proteins were measured. A total of 600 eggs from commercial breeding Cobb 50041 weeks, were selected by weight from 65 to $70 \mathrm{~g}$, were distributed on two incubators. A machine was kept at $4000 \mathrm{ppm}$ and the other to $3000 \mathrm{ppm} \mathrm{CO}_{2}$. We used a $2 \times 2$ factorial design. The hatchability was better to $3000 \mathrm{ppm}$ of $\mathrm{CO}_{2}$ and egg weight of $65 \mathrm{~g}$ chicken egg; the chicken was heavier with eggs of $70 \mathrm{~g}$, to more ppm of $\mathrm{CO}_{2}$ reduction in the loss of humidity, was observed over a large chicken, blood glucose levels were not affected, but the values of plasma protein were less than 3000 ppm $\mathrm{CO}_{2}$. Improved hatching parameters at lower ppm of $\mathrm{CO}_{2}$ during the incubation process.
\end{abstract}

Keywords: incubation, carbon dioxide, embryo, gases. 
DE desarrollo embrionario

$\mathrm{CO}_{2}$ dióxido de carbono

$\mathrm{pO}_{2} \quad$ presión parcial de oxígeno

$\mathrm{pCO}_{2}$
ABREVIATURAS

SA síndrome ascítico

HR humedad relativa

$\mathrm{O}_{2} \quad$ oxígeno

presión parcial de dióxido de carbono

\section{INTRODUCCIÓN}

El desarrollo embrionario (DE) depende en primera instancia de los poros del cascarón que permiten la difusión del oxígeno $\left(\mathbf{O}_{2}\right)$ y el dióxido de carbono $\left(\mathbf{C O}_{2}\right)$ entre el medio ambiente externo del huevo y la sangre del embrión (Cordeiro y Hincke, 2016). Este intercambio gaseoso se desarrolla a través de la membrana corioalantoidea (John, 2017), que está irrigada por vasos sanguíneos y cuya función es similar a la placenta de los fetos mamíferos (Koyama y Tennyson, 2016). La función primaria del sistema respiratorio, es transportar $\mathrm{O}_{2}$ y CO 2 , entre el ambiente y los tejidos (D'Alba et al., 2017); por lo que la respiración está regulada para enfrentar las demandas metabólicas, suministrando $\mathrm{O}_{2}$ y eliminando $\mathrm{CO}_{2}$ (Okur, 2019). La presión parcial de oxígeno $\left(\mathbf{p O}_{2}\right)$ y la presión parcial de dióxido de carbono $\left(\mathrm{pCO}_{2}\right)$ en la cámara de aire, son un estímulo para que el embrión realice el picaje (Deeming, 2016).

Gildersleeve y Boeschen (1983) realizaron un experimento con huevos de pavo, donde adicionaron niveles de $\mathrm{CO}_{2}$ más elevados que las concentraciones atmosféricas, con la finalidad de estimular el DE durante el inicio del periodo de incubación, aunque el DE se estimuló, no se encontraron diferencias en la incubabilidad entre los huevos incubados en rangos de $\mathrm{CO}_{2}$ atmosférico al $1 \%$ de $\mathrm{CO}_{2}$ durante los primeros 2 días de incubación, por lo que la temperatura del cascarón, junto a la concentración de $\mathrm{CO}_{2}$, afecta el peso corporal del pollo al nacer (Maatjens et al., 2014a; Maatjens et al., 2014b).

De Smit et al (2006) y De Smit et al (2008) modificaron las condiciones de ventilación, para elevar el $\mathrm{CO}_{2}$ durante los primeros 10 días del $\mathrm{DE}$, utilizando dos estirpes reproductoras, pesadas de 45 y 60 semanas; con diferentes niveles de susceptibilidad al síndrome ascítico (SA). Los niveles de $\mathrm{CO}_{2}$ resultaron en 1 y $1.5 \%$, así que el DE10 de niveles más altos de $\mathrm{CO}_{2}$ resultaron con mayor peso corporal absoluto y relativo (al peso del huevo) del DE10 hasta DE18; tuvieron un crecimiento acelerado, niveles elevados de corticosterona y $\mathrm{T} 3$ en plasma y $\mathrm{pCO}_{2}$ más alta en la cámara de aire; la ventana de eclosión fue más reducida en un tiempo de 10 a $15 \mathrm{~h}$, y el peso del pollo fue mayor con respecto a la ventilación normal. Asimismo García et al (2013), restringieron la ventilación durante los primeros 10 días del DE y encontraron una mejora en los parámetros de incubación.

Durante la última fase del desarrollo, el embrión de pollo varía su consumo de $\mathrm{O}_{2}$ con la temperatura ambiental (Deeming, 2016). Durante la $2^{\text {da }}$ fase del DE, una vez que el embrión produce su propio calor metabólico, elimina $\mathrm{CO}_{2}$ en un rango de 0.05 a $0.3 \%$ (D'Alba et al., 2017), por lo que también depende de la difusión de gases a través de los 
poros (Mortola y Labbe, 2005). Así que una condición de hipoxia puede limitar el DE, limitar el desarrollo del pico y patas, desarrollar hipertrofia del corazón, cambios en el ritmo cardiaco, edema pericárdico y pulmonar, cambios en la hemoglobina y SA; ya que en la última etapa del $\mathrm{DE}$, fase donde se produce la condición de hipoxia, el embrión llega a consumir hasta un 60\% más de $\mathrm{O}_{2}$ (Burggren y Elmonoufy, 2017; John, 2017; Itani et al., 2018). Otro factor que puede jugar un papel importante en el DE, es la altitud; ya que disminuye la conductancia del cascarón. En esta etapa el embrión necesita energía del metabolismo anaeróbico (Huang et al., 2017). Las reservas de glucosa en sangre y glucógeno tisular, son las que proporcionan esta energía, necesaria para realizar la eclosión (Fathollahipour et al., 2018). Se sabe que existen diferencias en el metabolismo de embriones con diferentes edades y líneas comerciales, por lo que si se pretende optimizar las condiciones de incubación, es necesario profundizar en sus requerimientos (Hamidu et al., 2018).

El objetivo de la presente investigación fue determinar el efecto de los parámetros de incubación con dos niveles de $\mathrm{CO}_{2}$ y dos pesos de huevo.

\section{MATERIAL Y MÉTODOS}

El experimento siguió las pautas institucionales y nacionales para el cuidado y uso de animales; todos los procedimientos fueron aprobados por el Comité de Revisión Ética de la Universidad de Colima. El estudio se realizó con 600 huevos fértiles de reproductora comercial Cobb 500, de 41 semanas de edad y peso de 65 y $70 \mathrm{~g}$. Los huevos se colocaron en dos incubadoras comerciales de una sola etapa (HatchTech; Gildetrom 25.3905 TB., Veenendaal, Netherlands), con capacidad de 4800 huevos/cada una. Las máquinas, las cuales tienen sensor de temperatura $\left( \pm 0 .{ }^{\circ} \mathrm{o} F\right)$, sensor de humedad $[ \pm 1$ $\%$ de humedad relativa $(\mathbf{H R})]$ y sensor de $\mathrm{CO}_{2}( \pm 100 \mathrm{ppm})$. Los huevos se voltearon a un ángulo de $45^{\circ}$ y luego $90^{\circ}$ cada hora.

La circulación del aire fue horizontal y laminar, a través de radiadores perforados, los cuales hacen que existan diferencias de presión, para una mejor distribución del aire y un flujo uniforme a través de cada masa de huevo, de arriba abajo y de adelante atrás. Las mismas máquinas hicieron la función de nacedoras, y en ambas se realizó el manejo de rutina propio de una planta incubadora, desde la recepción del huevo, hasta el retiro de las máquinas nacedoras.

\section{Diseño experimental}

El experimento se estableció como un diseño completamente al azar, con arreglo factorial $2 \times 2$, con 2 concentraciones de $\mathrm{CO}_{2}$ de la incubadora (3000 vs. 4000 ppm), y 2 pesos de huevo (65 y $70 \mathrm{~g}$ ); los tratamientos se dividieron en 2 grupos posteriores con 2 tratamientos por grupo. De manera previa, se realizó una selección donde se descartaron huevos rotos, sucios, microfracturados, deformes y fuera de peso. Los huevos se identificaron de forma individual, con marcador de tinta indeleble sobre la superficie ancha donde se localiza la cámara de aire. Las máquinas incubadoras se mantuvieron a igual 
temperatura por etapa durante todo el proceso de incubación; de $37.8^{\circ} \mathrm{C}$ durante el día $0,37.6{ }^{\circ} \mathrm{C}$ del día 1 al $8,37.5^{\circ} \mathrm{C}$ del día 9 al 11 y $37.2^{\circ} \mathrm{C}$ del día 12 al 21 . La HR se mantuvo en $50 \%$ durante todo el proceso de incubación. La concentración de $\mathrm{CO}_{2}$ fue monitoreada durante todo el proceso de incubación, para verificar que se mantuviera dentro de los rangos establecidos en el protocolo de investigación.

Los criterios de respuesta fueron peso individual de los huevos antes de incubar y durante la transferencia al día 18, para determinar por diferencia de peso la pérdida de humedad, incubabilidad, peso corporal y tamaño (medición del pico hasta el dedo medio sin considerar la uña).

\section{Mediciones de parámetros sanguíneos}

Se asignaron al azar 20 embriones para su uso en la determinación de parámetros sanguíneos. La sangre se extrajo de la vena yugular de los embriones o pollos, con una jeringa de $1 \mathrm{~mL}$ y una aguja de calibre 30 , y se recogió en tubos heparinizados. Posteriormente, se extrajo sangre en un capilar heparinizado $(150 \mu \mathrm{L})$ y se presentó inmediatamente a un analizador de gases en sangre (GEM Premier 3000; Instrumentation Laboratory., Lexington, Massachusetts), para determinar los criterios de respuesta glucosa, hematocrito y proteínas plasmáticas.

\section{Análisis estadístico}

Los datos se procesaron utilizando el programa estadístico (SAS, System, v. 8.2, Cary, NC). Se examinaron las distribuciones de las medias y los residuos para verificar los supuestos del modelo. Los valores de incubabilidad, peso del pollo, pérdida de humedad, tamaño del pollo, glucosa, hematocrito y proteínas plasmáticas se analizaron por momento de muestreo; utilizando regresión lineal general (PROC GLM), con 2 concentraciones de $\mathrm{CO}_{2}, 2$ pesos de huevo y su interacción como variables de clase. Para todos los parámetros, se consideró pollito como la unidad experimental. Se realizó una prueba de comparación múltiple de Tukey, cuando se encontró que el efecto grupo era significativo $(P<0.05)$. Los datos expresados en porcentajes fueron transformados a la proporción arco seno para su análisis.

\section{RESULTADOS}

En la tabla 1, se observa que el mayor porcentaje de incubabilidad fue con la concentración a 3000 ppm de $\mathrm{CO}_{2}$, y con $65 \mathrm{~g}$ en el peso de los huevos. Los niveles de $\mathrm{CO}_{2}$ considerados en el experimento no afectaron el peso del pollo; por lo que los tratamientos con huevos de $70 \mathrm{~g}$ obtuvieron pollos más pesados. El menor porcentaje en la pérdida de humedad con respecto a la concentración de $\mathrm{CO}_{2}$, se registró a 4000 ppm y sin diferencias estadísticas $(P<0.05)$, entre huevos con 65 y $70 \mathrm{~g}$. El mayor tamaño del pollo con respecto a la concentración de $\mathrm{CO}_{2}$, se observó a 3000 ppm y sin diferencias estadísticas entre huevos con 65 y $70 \mathrm{~g}$. 
Tabla 1. Efectos de 2 concentraciones de dióxido de carbono de la incubadora (3000 y 4000 ppm) y 2 pesos de huevo (65 y $70 \mathrm{~g}$ ) sobre incubabilidad, peso del pollo, pérdida de humedad, tamaño del pollo, glucosa, hematocrito y proteínas plasmáticas $\left(n=20\right.$ por $\mathrm{CO}_{2} \times$ peso del huevo)

\begin{tabular}{|c|c|c|c|c|c|c|c|}
\hline Factor & $\begin{array}{c}\text { Incubabilidad } \\
(\%)\end{array}$ & $\begin{array}{l}\text { Peso del } \\
\text { pollo }(\mathrm{g})\end{array}$ & $\begin{array}{c}\text { Pérdida de } \\
\text { humedad (\%) }\end{array}$ & $\begin{array}{l}\text { Tamaño del } \\
\text { pollo }(\mathrm{cm})\end{array}$ & $\begin{array}{l}\text { Glucosa } \\
\text { (mg/dL) }\end{array}$ & $\begin{array}{c}\text { Hematocrito } \\
(\%)\end{array}$ & $\begin{array}{c}\text { Proteínas } \\
\text { plasmáticas }(\mathrm{g} / \mathrm{dL})\end{array}$ \\
\hline \multicolumn{8}{|l|}{$\mathrm{CO}_{2}(\mathrm{ppm})$} \\
\hline 3000 & $88.10^{a}$ & 46.53 & $10.19^{a}$ & $19.44^{a}$ & 200.05 & 33.60 & $2.43^{b}$ \\
\hline 4000 & $86.40^{\mathrm{b}}$ & 47.00 & $8.70^{\mathrm{b}}$ & $18.44^{b}$ & 188.85 & 36.80 & $2.71^{\mathrm{a}}$ \\
\hline Error de cuadrado medio & 2.77 & 2.91 & 2.59 & 0.22 & 373.53 & 12.05 & 0.17 \\
\hline \multicolumn{8}{|l|}{ Peso del huevo $(\mathrm{g})$} \\
\hline 65 & $88.10^{a}$ & $44.99^{b}$ & 9.49 & 18.83 & 195.20 & 34.60 & 2.56 \\
\hline 70 & $86.40^{\mathrm{b}}$ & $48.54^{a}$ & 9.41 & 19.06 & 193.70 & 35.80 & 2.59 \\
\hline Error de cuadrado medio & 2.77 & 2.91 & 2.59 & 0.22 & 373.53 & 12.05 & 0.17 \\
\hline \multicolumn{8}{|l|}{$\mathrm{CO}_{2}(\mathrm{ppm}) \times$ peso del huevo $(\mathrm{g})$} \\
\hline $3000 \times 70$ & 87.60 & 48.93 & 9.87 & 19.45 & 204.50 & 32.90 & 2.53 \\
\hline $4000 \times 65$ & 87.60 & 45.85 & 8.47 & 18.22 & 194.80 & 34.90 & 2.78 \\
\hline $4000 \times 70$ & 85.20 & 48.16 & 8.94 & 18.67 & 182.90 & 38.70 & 2.65 \\
\hline \multicolumn{8}{|l|}{$P$-valor } \\
\hline $\mathrm{CO}_{2}$ & 0.03 & 0.38 & $<.0001$ & $<.0001$ & 0.07 & 0.006 & 0.04 \\
\hline Peso del huevo & 0.03 & $<.0001$ & 0.62 & 0.13 & 0.80 & 0.28 & 0.82 \\
\hline $\mathrm{CO}_{2} \times$ peso del huevo & 0.36 & 0.02 & 0.0007 & 0.15 & 0.09 & 0.02 & 0.23 \\
\hline
\end{tabular}

a,bLas medias de los mínimos cuadrados seguidas de diferentes superíndices dentro de una columna y factor son significativamente diferentes $(P<0.05)$. 
En la tabla 1, se observa que la combinación entre las concentraciones de $\mathrm{CO}_{2}$ y los pesos del huevo, no mostraron diferencias dentro de grupo (65 y $70 \mathrm{~g}$, respectivamente); pero sí entre grupos, con el menor peso del pollo $(P<0.05)$, en el tratamiento que combina $3000 \mathrm{ppm}$ de $\mathrm{CO}_{2}$ con huevos de $65 \mathrm{~g}$. Con respecto al tamaño del pollo, el resultando con mayor talla se observó en la combinación de 3000 ppm de $\mathrm{CO}_{2}$, con huevos de 65 y $70 \mathrm{~g}$. La concentración de glucosa sanguínea no mostró diferencias en ambos factores. El mayor porcentaje de hematocrito con respecto a la concentración de $\mathrm{CO}_{2}$, se registró a 4000 ppm y sin diferencias estadísticas $(P<0.05)$, entre huevos con 65 y $70 \mathrm{~g}$. La mayor concentración de proteínas plasmáticas con respecto a la concentración de $\mathrm{CO}_{2}$, se registró a 4000 ppm. El resultando con mayor porcentaje de hematocrito se registró en la combinación de 4000 ppm de $\mathrm{CO}_{2}$, con huevos de $70 \mathrm{~g}$.

\section{DISCUSIÓN}

La incubabilidad (es la capacidad del huevo para eclosionar), mejora con una menor concentración de $\mathrm{CO}_{2}$ (Fathollahipour et al., 2018). En el presente trabajo se encontró que la concentración más baja de $\mathrm{CO}_{2}$ mejora la incubabilidad; por lo que la integridad del cascarón juega un papel importante para el intercambio gaseoso; así que cuando el embrión llega al día 18 de incubación, inicia la respiración pulmonar, donde la hormona tiroidea juega un rol importante en el desarrollo del surfactante pulmonar (Hamidu et al., 2018). El proceso de la respiración pulmonar aporta $\mathrm{O}_{2}$ al embrión del pollo (Deeming, 2016); sin embargo, la maduración de la respiración pulmonar sin tener el cascarón intacto, y con el incremento de la demanda metabólica de $\mathrm{O}_{2}$ al final de la incubación, ocasionan un aumento en la $\mathrm{pCO}_{2}$ y una disminución en la $\mathrm{pO}_{2}$ en la cámara de aire, (Flores-Santin et al., 2018), activando el mecanismo detonador del nacimiento (Ramachandran y McDaniel, 2018). Así que el DE normal del pollo, depende de los cambios de aire que se realizan a través de la membrana corioalantoidea (John, 2017), que junto con los poros del cascarón realiza los intercambios de $\mathrm{O}_{2}$ y $\mathrm{CO}_{2}$ entre la sangre y el medio ambiente (Deeming, 2016).

El cascarón es el principal responsable de la diferencia entre la $\mathrm{pCO}_{2}$ y el vapor de agua, donde el flujo de $\mathrm{O}_{2}$ es afectado por el cascarón y la membrana interna (Ramachandran y McDaniel, 2018); de manera que puede ser una de las razones principales por la que la incubabilidad no fue efectiva a mayor nivel de $\mathrm{CO}_{2}$. De la misma manera la conductancia del gas en el cascarón, depende de la proporción de área del poro por su longitud; al final esta relación es equivalente al grosor del cascarón (Cordeiro y Hincke, 2016).

En el presente estudio, se observó que al disponer de una menor concentración de $\mathrm{CO}_{2}$ en el ambiente, se mejoró la eclosión; ya que no se creó una condición de hipoxia al final del proceso de incubación y no se afectó la $\mathrm{pO}_{2}$ y la $\mathrm{pCO}_{2}$ en la cámara de aire. Cuando los embriones se someten a condiciones de hipoxia durante el proceso de incubación, se inhibe el crecimiento del cuerpo, pico y patas; tal como lo mencionan (Burggren y 
Elmonoufy, 2017). En el experimento, el peso del pollo no se afectó por las diferentes concentraciones de $\mathrm{CO}_{2}$, pero sí fueron más pesados los pollos que provenían de huevos con $70 \mathrm{~g}$. Al respecto, se sabe que el peso del pollo está directamente relacionado con el peso del huevo (D'Alba et al., 2017). El tamaño del pollo fue mayor cuando la concentración de $\mathrm{CO}_{2}$ fue de 3000 ppm, estos datos sugieren que si los embriones se desarrollan en condiciones ambientales con una concentración baja de $\mathrm{O}_{2}$, pierden peso durante el picaje y la eclosión (Ramachandran y McDaniel, 2018). Por lo tanto, los embriones en condiciones densas de $\mathrm{O}_{2}$ ambiental ganan peso; esto implica que los embriones en escenarios ambientales ricos en $\mathrm{O}_{2}$, están mejor preparados metabólicamente para iniciar el picaje y el nacimiento (Deeming, 2016).

En el estudio, el tamaño del pollo se utilizó como criterio de calidad para determinar el desempeño productivo de la parvada; por otro lado, el contenido de glucógeno en los tejidos corporales, es importante en el requerimiento fisiológico al momento de iniciar el picaje (Maatjens et al., 2014a; Maatjens et al., 2014b). De igual manera, influye directamente en la sobrevivencia embrionaria; así, en el estudio las condiciones de $\mathrm{CO}_{2}$ no afectaron los niveles de glucosa sanguínea en los pollos, lo cual puede condicionar a la vitalidad de las aves en la granja.

El nivel de hematocrito mostró una tendencia numérica ligeramente mayor en el tratamiento con más concentración de $\mathrm{CO}_{2}$, información similar a lo reportado por Scheele et al. (2003) quienes cuantificaron valores elevados de hematocrito como resultado de un incremento en la respuesta eritropoyética. La concentración de proteínas plasmáticas, fue menor cuando las condiciones de $\mathrm{CO}_{2}$ se mantuvieron en 4000 ppm; estos datos sugieren que en ambientes ricos en $\mathrm{CO}_{2}$, se utiliza más la respuesta eritropoyética (Ramachandran y McDaniel, 2018), y como consecuencia se reduce la concentración de proteínas plasmáticas, tal como se observó en el presente estudio.

Durante el DE del pollo, las incubadoras comerciales manejan concentraciones de 4000 ppm de $\mathrm{CO}_{2}$; pero con los resultados obtenidos, se sugiere bajar dicha concentración a 3000 ppm, para mejorar principalmente la incubabilidad.

\section{CONCLUSIÓN}

Concentraciones de 3000 ppm de $\mathrm{CO}_{2}$ mejoran la incubabilidad, pérdida de humedad y el tamaño del pollo; asimismo tiene niveles bajos de proteínas plasmáticas.

\section{LITERATURA CITADA}

BURGGREN WW, Elmonoufy NA. 2017. Critical developmental windows for morphology and hematology revealed by intermittent and continuous hypoxic incubation in embryos of quail (Coturnix coturnix). PLoS One. 12(9):e0183649. ISSN: 1932-6203. http://dx.doi.org/10.1371/journal.pone.0183649 
CORDEIRO CM, Hincke MT. 2016. Quantitative proteomics analysis of eggshell membrane proteins during chick embryonic development. Journal of Proteomics. 130:1125. ISSN: 1876-7737. http://dx.doi.org/10.1016/j.jprot.2015.08.014

D'ALBA L, Torres R, Waterhouse GIN, Eliason C, Hauber ME, Shawkey MD. 2017. What does the eggshell cuticle do? a functional comparison of avian eggshell cuticles. Physiological and Biochemical Zoology. 90(5):588-599. ISSN: 1537-5293. http://dx.doi.org/10.1086/693434

DEEMING DC. 2016. How does the bird-nest incubation unit work? Avian Biology Research. 9(2):103-113. ISSN: 1758-1559. http://dx.doi.org/10.3184/175815516X14567543242701

DE SMIT L, Bruggeman V, Tona JK, Debonne M, Onagbesan O, Arckens L, De Baerdemaeker J, Decuypere E. 2006. Embryonic developmental plasticity of the chick: Increased $\mathrm{CO}_{2}$ during early stages of incubation changes the developmental trajectories during prenatal and postnatal growth. Comparative Biochemistry and Physiology Part A: Molecular \& Integrative Physiology. 145(2):166-175. ISSN: 1095-6433. https://doi.org/10.1016/j.cbpa.2006.06.046

DE SMIT L, Bruggeman V, Debonne M, Tona JK, Kamers B, Everaert N, Witters A, Onagbesan O, Arckens L, De Baerdemaeker J, Decuypere E. 2008. The effect of nonventilation during early incubation on the embryonic development of chicks of two commercial broiler strains differing in ascites susceptibility. Poultry Science. 87(1):551560. ISSN: 0032-5791. https://doi.org/10.3382/ps.2007-00322

FATHOLLAHIPOUR S, Patil PS, Leipzig ND. 2018. Oxygen regulation in development: lessons from embryogenesis towards tissue engineering. Cells Tissues Organs. 205(56):350-371. ISSN: 1422-6421. http://dx.doi.org/10.1159/000493162

FLORES-SANTIN J, Rojas Antich M, Tazawa H, Burggren WW. 2018. Hematology from embryo to adult in the bobwhite quail (Colinus virginianus): Differential effects in the adult of clutch, sex and hypoxic incubation. Comparative biochemistry and physiology. Part $A$, Molecular \& integrative physiology. 218(1):24-34. ISSN: 0301-5092. http://dx.doi.org/10.1016/j.cbpa.2018.01.005

GARCÍA HJ, Juárez EMA, Córdova SL. 2013. Gradual increase of $\mathrm{CO}_{2}$ during first stages of incubation with late change of $\mathrm{O}_{2}$ partial pressure, modifies the hatch trajectory of broiler chicks. Veterinaria México. 44(1):1-16. ISSN: 0032-5791.

http://veterinariamexico.unam.mx/index.php/vet/article/view/325 
GILDERSLEEVE RP, Boeschen DP. 1983. The effects of incubator carbon dioxide level on turkey hatchability. Poultry science. 62(5):779-784. ISSN: 0032-5791. http://dx.doi.org/10.3382/ps.0620779

HAMIDU JA, Torres CA, Johnson-Dahl ML, Korver DR. 2018. Physiological response of broiler embryos to different incubator temperature profiles and maternal flock age during incubation. 1. Embryonic metabolism and day-old chick quality. Poultry Science. 97(8):2934-2946. ISSN: 1525-3171. http://dx.doi.org/10.3382/ps/pey089

HUANG S, Zhang L, Rehman MU, lqbal MK, Lan Y, Mehmood K, Zhang H, Qiu G, Nabi F, Yao W, Wang M, Li J. 2017. High altitude hypoxia as a factor that promotes tibial growth plate development in broiler chickens. PLoS One. 12(3):e0173698. ISSN: 1932-6203. http://dx.doi.org/10.1371/journal.pone.0173698

ITANI N, Salinas CE, Villena M, Skeffington KL, Beck C, Villamor E, Blanco CE, Giussani DA. 2018. The highs and lows of programmed cardiovascular disease by developmental hypoxia: studies in the chicken embryo. Journal of Physiology. 596(15):2991-3006. ISSN: 1469-7793. http://dx.doi.org/10.1113/JP274111

JOHN NM. 2017. Structure and function of the shell and the chorioallantoic membrane of the avian egg: embryonic respiration. In: The Biology of the Avian Respiratory System. Springer. 219-247 p. ISBN: 978-3-319-44152-8. https://doi.org/10.1007/978-3-31944153-5_9

KOYAMA T, Tennyson AJD. 2016. Respiratory pores on Ostrich Struthio camelus (Aves: Struthionidae) eggshells. Advances in Experimental Medicine and Biology. 923(1):51-55. ISSN: 0065-2598. http://dx.doi.org/10.1007/978-3-319-38810-6_7

MAATJENS CM, Reijrink IA, Molenaar R, van der Pol CW, Kemp B, van den Brand H. 2014a. Temperature and $\mathrm{CO}_{2}$ during the hatching phase. I. Effects on chick quality and organ development. Poultry science. 93(3):645-654. ISSN: 0032-5791. http://dx.doi.org/10.3382/ps.2013-03490

MAATJENS CM, Reijink IA, van den Anker I, Molenaar R, van der Pol CW, Kemp B, van den Brand $\mathrm{H}$. 2014b. Temperature and $\mathrm{CO}_{2}$ during the hatching phase. II. Effects on chicken embryo physiology. Poultry science. 93(3):655-663. ISSN: 0032-5791. http://dx.doi.org/10.3382/ps.2013-03491 
MORTOLA JP, Labbe K. 2005. Oxygen consumption of the chicken embryo: interaction between temperature and oxygenation. Respiratory physiology \& neurobiology. 146(1):97-106. ISSN: 1569-9048. http://dx.doi.org/10.1016/j.resp.2004.10.011

OKUR N. 2019. Effects of incubator carbon dioxide and oxygen levels, and egg weight on Broilers' hatchability of fertile eggs. Brazilian Journal of Poultry Science. 21(3). ISSN: 1516-635X. http://dx.doi.org/10.1590/1806-9061-2019-1038

RAMACHANDRAN R, McDaniel CD. 2018. Parthenogenesis in birds: a review. Reproduction. 155(6):R245-R257. ISSN: 1741-7899. http://dx.doi.org/10.1530/REP-170728

SAS. 2001. SAS/STAT User's guide. 8.2, v. SAS Institute Inc. Cary, NC.

SCHEELE CW, van Der Klis JD, Kwakernaak C, Buys N, Decuypere E. 2003. Haematological characteristics predicting susceptibility for ascites. 2. High haematocrit values in juvenile chickens. British Poultry Science. 44(3):484-489. ISSN: 0007-1668. http://dx.doi.org/10.1080/00071660310001598300 